\title{
Functional Connectivity Correlates of Reduced Goal- directed Behaviors in Behavioural Variant Frontotemporal Dementia
}

Valérie Godefroy ( $\sim$ vlrgodefroy@gmail.com )

Institut du cerveau et de la moelle epiniere https://orcid.org/0000-0001-8330-5413

Bénédicte Batrancourt

Institut du cerveau et de la moelle epiniere

Sylvain Charron

Institut de Psychiatrie et Neurosciences de Paris

Arabella Bouzigues

Institut du cerveau et de la moelle epiniere

David Bendetowicz

Institut du cerveau et de la moelle epiniere

Guilhem Carle

APHP: Assistance Publique - Hopitaux de Paris

Armelle Rametti-Lacroux

Institut du cerveau et de la moelle epiniere

Stéphanie Bombois

APHP: Assistance Publique - Hopitaux de Paris

Emmanuel Cognat

GH Lariboisiere Fernand-Widal: Groupe Hospitalier Saint-Louis Lariboisiere et Fernand-Widal

Raffaella Migliaccio

Institut du cerveau et de la moelle epiniere

Richard Levy

Institut du cerveau et de la moelle epiniere

\section{Research Article}

Keywords: frontotemporal dementia, apathy, goal-directed behavior, ecological design, functional connectivity, resting state

Posted Date: January 25th, 2022

DOl: https://doi.org/10.21203/rs.3.rs-1279408/v1 
License: (c) (i) This work is licensed under a Creative Commons Attribution 4.0 International License. Read Full License 


\section{Functional connectivity correlates of reduced goal-directed behaviors in}

\section{behavioural variant frontotemporal dementia}

Valérie Godefroy ${ }^{1}$, Bénédicte Batrancourt ${ }^{1}$, Sylvain Charron $^{2,3}$, Arabella Bouzigues ${ }^{1}$, David Bendetowicz ${ }^{1,4,5}$, Guilhem Carle ${ }^{5}$, Armelle Rametti-Lacroux ${ }^{1}$, Stéphanie Bombois ${ }^{4}$, Emmanuel Cognat ${ }^{6,7}$, Raffaella Migliaccio ${ }^{1,4,5}$, Richard Levy ${ }^{1,4,5}$

\section{Author affiliations:}

(1) Inserm U 1127, CNRS UMR 7225, Sorbonne Université, Institut du Cerveau - Paris Brain Institute - ICM, Inserm, CNRS, AP-HP, Hôpital de la Pitié Salpêtrière, Paris, France

(2) INSERM U1266, Institut de Psychiatrie et Neurosciences de Paris, Université de Paris, Paris, France

(3) Department of Neuroradiology, Hôpital Sainte-Anne, Université de Paris, Paris, France

(4) Department of Neurology, IM2A, AP-HP, Groupe Hospitalier Pitié-Salpêtrière, Paris, France

(5) Behavioural Neuropsychiatry Unit, AP-HP, Hôpital de la Salpêtrière, Paris, France

(6) UMRS 1144, INSERM, F-5010, Université de Paris, Paris, France

(7) Centre de Neurologie Cognitive, Hôpital Lariboisière Fernand-Widal, APHP Nord, F75010, Paris, France

Correspondence to: Valérie Godefroy

Inserm U 1127, CNRS UMR 7225, Sorbonne Université, Institut du Cerveau - Paris Brain Institute - ICM, Inserm, CNRS, AP-HP, Hôpital de la Pitié Salpêtrière

47 Boulevard de l'Hôpital, 75013 Paris, France

E-mail: vlrgodefroy@gmail.com 


\section{Acknowledgments}

Valérie Godefroy is supported by Malakoff Médéric Humanis company. Arabella Bouzigues is supported by "Fondation Vaincre Alzheimer". Raffaella Migliaccio is supported by France Alzheimer and Philippe Chatrier Foundations, and by Rosita Gomez association.

We sincerely acknowledge the participants and caregivers for their involvement in this study. 


\begin{abstract}
INTRODUCTION: We explored the resting state functional connectivity correlates of apathy assessed as a multidimensional construct, using behavioral metrics, in behavioral variant frontotemporal dementia (bvFTD).
\end{abstract}

METHODS: We recorded the behavior of 20 bvFTD patients and 16 healthy controls (HC) in a close-to-real-life situation including a free phase ( $\mathrm{FP}$ - in which actions were self-initiated) and a guided phase (GP - in which initiation of actions was facilitated by external guidance). We investigated the activity time and walking episode features as quantifiers of apathy. We used the means $((\mathrm{FP}+\mathrm{GP}) / 2)$ and the differences $(\mathrm{FP}-\mathrm{GP})$ calculated for these metrics as well as measures by questionnaires to extract apathy dimensions by factor analysis. We assessed two types of connectivity measures (local low-frequency signal power and distant seed-based functional connectivity) and explored their relationship with extracted apathy dimensions.

RESULTS: Apathy in bvFTD was associated with lower time spent in activity combined with walking episodes of higher frequency, lower acceleration and higher duration. Using these behavioral metrics and apathy measures by questionnaires, we disentangled two dimensions: the global reduction of goal-directed behaviors and the specific deficit of selfinitiation. Global apathy was associated with lower resting state activity within prefrontal cortex and lower connectivity of salience network hubs while the decrease in self-initiation was related to increased connectivity of parietal default-mode network hubs.

DISCUSSION: Through a new dimensional approach of apathy, we dissociated the functional connectivity correlates of global apathy and self-initiation deficit. This contributes to better understand the role of functional networks in the production of goal-directed behaviors. 
Keywords: frontotemporal dementia; apathy; goal-directed behavior; ecological design; functional connectivity; resting state

Abbreviations: $\mathrm{AAD}=$ auto-activation deficit; $\mathrm{AD}=$ Alzheimer's disease; $\mathrm{ACC}=$ anterior cingulate cortex; $\mathrm{AI}=$ anterior insula; $\mathrm{bvFTD}=$ behavioural variant frontotemporal dementia; DAS $=$ Dimensional Apathy Scale; DLPFC $=$ dorsolateral prefrontal cortex; DMN: Default Mode Network; DRS = Mattis Dementia Rating Scale; EFA = Exploratory Factor Analysis; $\mathrm{FAB}=$ Frontal Assessment Battery; fALFF = fractional amplitude of low-frequency fluctuations; FP = Free (or self-guided) Phase; FTD = frontotemporal dementia; GP = Guided (or hetero-guided) Phase; $\mathrm{HC}=$ healthy controls; $\mathrm{LPC}=$ lateral parietal cortex; $\mathrm{MCC}=$ midcingulate cortex; MMSE $=$ Mini-Mental State Evaluation; $\mathrm{MPFC}=$ medial prefrontal cortex; PACT $=$ Philadelphia Apathy Computerized Test; OFC $=$ orbitofrontal cortex; PCC $=$ posterior cingulate cortex; $\mathrm{PFC}=$ prefrontal cortex; $\mathrm{ROI}=$ region of interest; $\mathrm{SAS}=$ Starkstein Apathy Scale; SBC = seed-based connectivity; SFG = superior frontal gyrus; SMA = supplementary motor area; $\mathrm{SN}=$ Salience Network; VBM = Voxel-Based Morphometry; $\mathrm{WEp}=$ walking episodes 


\section{BACKGROUND}

Apathy is the most frequent behavioral syndrome in neurological and psychiatric diseases (Levy and Dubois 2006; Levy 2012; Le Heron et al. 2018) and is also prevalent in varying degrees in healthy people (Ang et al. 2017). In neurological diseases, apathy is considered as a major source of morbidity predicting bad prognosis (Levy 2012; Lansdall et al. 2019; Malpetti et al. 2021). Apathy is also associated with a higher level of global functional impairment and loss of autonomy (Lechowski et al. 2009; Wadsworth et al. 2012), and it has a negative impact on the quality of life of both patients and their caregivers (Hurt et al. 2008). Considering the globally high prevalence of apathy and the extent of its debilitating consequences, it is of high importance to advance our understanding of this syndrome with the final aim of improving its treatment.

Apathy is traditionally defined as a "lack of motivation not attributable to diminished level of consciousness, cognitive impairment or emotional distress'” (Marin 1991, 1996). The clinical scales typically used to assess apathy (e.g., the Starkstein Apathy Scale - SAS (Starkstein et al. 1992)) are based on this definition and mostly rely on questions about a patient's internal state of mind. They are therefore biased by the subjective perspective of the reporter (Levy and Dubois 2006; Levy 2012; Godefroy et al. 2020). In line with the recent international consensus on apathy criteria in neurodegenerative diseases (Robert et al. 2018), we define apathy as an observable quantitative reduction of goal-directed activity. For the present study, relying on this behavioral definition of apathy, we have developed new tools allowing us to objectively quantify goal-directed behaviors in semi-ecological conditions. This was a starting point towards a better understanding of the mechanisms involved in the quantitative production of goal-directed behaviors.

Several non-exclusive mechanisms may underlie the reduction of goal-directed behaviors observed in apathetic states (Massimo et al. 2014). Levy and Colleagues (Levy and 
Dubois 2006; Levy 2012; Godefroy et al. 2020) have postulated the existence of three subtypes of apathy mechanisms (i.e., 'emotional-affective', 'cognitive', and 'auto-activation') corresponding to the disruption of different processes underpinned by distinct neural substrates. The 'emotional-affective' subtype corresponds to the disruption of emotional processing which may disrupt the motivation for goal-directed behaviors due to emotional desensitization to both positive and negative stimuli. In the 'cognitive' apathy subtype, goaldirected behaviors are reduced due to impaired cognitive functions needed to elaborate and implement a plan of actions. The 'auto-activation' subtype refers to difficulties in activating thoughts or initiating the motor program necessary to complete the behavior. All the tools developed to assess apathy as a multidimensional construct, like the Philadelphia Apathy Computerized Test (PACT) (Massimo et al. 2015) or the Dimensional Apathy Scale (DAS) (Radakovic and Abrahams 2014), dissociate these three aspects of apathy although they are potentially concurrent and overlapping (Ducharme et al. 2018). Besides, several authors (Levy and Dubois 2006; Levy 2012; Ducharme et al. 2018; Le Heron et al. 2018; Godefroy et al. 2020) agree on the assumption that a deficit of self-initiation of goal-directed behaviors, potentially reversed by hetero-activation, is a central feature to explain the global reduction of goal-directed behaviors observed in the apathy syndrome. This type of deficit can be illustrated by the case of patients with bilateral basal ganglia lesions resulting in a syndrome of auto-activation deficit (AAD) (Laplane and Dubois 2001). In these patients, despite the loss of self-initiated behaviors, the ability to execute externally-driven behavior is relatively spared (Schmidt et al. 2008).

Neurodegenerative dementias are very interesting lesional models to investigate the neural correlates of apathy, in particular within the frontotemporal dementia (FTD) spectrum (including the behavioral variant $[\mathrm{bvFTD}]$ and other language variants). These can serve as a model to understand apathy in other neuropsychiatric disorders. In bvFTD, the presence of 
apathy is one of the main and most frequent criteria enabling a clinical diagnosis of bvFTD (Rascovsky et al. 2007, 2011) and it remains almost constant throughout the disease (Pasquier et al. 1999). According to a literature review (Ducharme et al. 2018), apathy in FTD is most robustly associated with atrophy, hypometabolism and/or hypoperfusion in the dorsolateral prefrontal cortex (DLPFC), the anterior and middle cingulate cortex (ACC and MCC), the orbitofrontal cortex (OFC) and the medial superior frontal gyrus (SFG). However, most studies that have investigated the neural correlates of apathy in FTD assessed apathy as a unidimensional construct using clinical scales. Some (Massimo et al. 2015; Kumfor et al. 2018; Wei et al. 2020), but this literature remains sparse, have explored the neural bases of apathy as a multidimensional concept. Moreover, to our knowledge, only three studies (Day et al. 2013; Farb et al. 2013; Zhou et al. 2020) related functional connectivity with apathy (measured as a unidimensional concept) in FTD patients. Functional connectivity is defined as the temporal dependency of neuronal activation patterns of anatomically separated brain regions driven by low-frequency fluctuations (from .01 to $.1 \mathrm{~Hz}$ ) in the blood oxygen-level dependent (BOLD) signal (van den Heuvel and Hulshoff Pol 2010). The exploration of resting state functional connectivity bears great potential in patients with dementia in particular to explain their neuropsychiatric symptoms (Zhou and Seeley 2014).

In this study, to address the issue of using only subjective measures of apathy by clinical scales, we firstly aimed to identify objective behavioral markers associated with apathy in bvFTD using an ecological approach. We used an original paradigm capturing participants' behavior (through sensor and video recording) in a close-to-real-life situation and we investigated the hypothesis that several behavioral metrics could be used as quantifiers of goal-directed behaviors contributing to assess apathy. In this particular ecological setting, we assumed that the total time spent in goal-directed activity and characteristics of walking while 
moving in the room could be such quantifiers of goal-directed behaviors. Indeed, the selected characteristics of walking episodes were supposed to reflect the extent to which movements in the room were oriented towards specific goals (instead of being mere wanderings).

The second aim of this study was to tackle another issue related to the assessment of apathy. As the deficit of self-initiation of goal-directed behaviors is theorized to be one of the central mechanisms of apathy, disentangling the assessment of the global reduction of goaldirected behaviors (in other words, the severity of apathy syndrome) from the assessment of the specific self-initiation deficit (in other words, the subtype of apathy as a unique dimension) could be useful. Our ecological paradigm of behavior tracking included both a 'free phase' (FP) in which goal-directed behaviors were totally self-initiated and a 'guided phase' (GP) in which initiation was facilitated by hetero-guidance. Thus, we could calculate for each quantifier of goal-directed behaviors: first, the mean on the two phases as a potential indicator of the global reduction of goal-directed behaviors; second, the difference between the two phases as a potential indicator of the specific deficit of self-initiation. Indeed, the more participants are characterized by a specific deficit of self-initiation underlying apathy, the more their markers of goal-directed behaviors should improve in GP compared to FP.

The third objective of the study responded to the need to further investigate the functional connectivity correlates of apathy in bvFTD. For this purpose, we used the two dimensions of apathy (global apathy and specific self-initiation deficit), which we related to two complementary measures of resting state connections: a measure of local low-frequency signal power explored at the whole-brain level and a measure of distant functional connectivity between several regions of interest (ROI) and the rest of the brain. We selected ROI of the salience and default-mode networks, according to a model of connectivity changes in bvFTD patients suggested by Zhou, Seeley and colleagues (Zhou et al. 2010; Zhou and Seeley 2014). According to these authors, evidence to date suggests that bvFTD mainly 
targets the salience network (SN), beginning in central nodes which activate in response to diverse emotionally significant internal and external stimuli or conditions. These nodes include the anterior insula (AI), considered as an afferent salience structure, and the anterior cingulate cortex (ACC), considered as an efferent visceromotor region. Moreover, upon initiation of a goal-directed behavior, a uniform pattern of deactivation has been observed in healthy adults across a network of brain regions that demonstrate a high degree of functional connectivity including: the precuneus/ posterior cingulate cortex (PCC), medial prefrontal cortex (MPFC), and bilateral parietal cortices (LPC) (Raichle et al. 2001). These brain regions make up the main hubs of the default-mode network (DMN), a network supporting a "default mode" of brain function when an individual is awake and alert but not actively involved in a goal-directed task (Raichle et al. 2001). Building on the results of a study investigating functional network connectivity patterns in Alzheimer's disease (AD) and bvFTD patients, Zhou and Colleagues suggested that the clinical severity of bvFTD symptoms was associated with reduced SN connectivity but enhanced DMN connectivity (Zhou et al. 2010).

\section{MATERIAL AND METHODS}

\subsection{Participants and protocol overview}

Twenty bvFTD patients and $16 \mathrm{HC}$, matched to patients for age, gender and education level were included in this study. BvFTD patients were recruited in two tertiary referral centers, at the Pitié-Salpêtrière Hospital and the Lariboisière Fernand-Widal Hospital, in Paris. They were diagnosed according to the International Consensus Diagnostic Criteria (Rascovsky et al. 2011). To respect inclusion criteria, bvFTD patients had to present a Mini-Mental State Evaluation (MMSE) score of at least 20 (to make sure that they were able to undergo the full 
protocol). Healthy controls (HC) were recruited by public announcement. They had to present a MMSE score of at least 27.

The data gathered for this article are part of the ECOCAPTURE protocol (Clinicaltrials.gov:NCT02496312; see Batrancourt et al. (Batrancourt et al. 2019) and Supplementary file 1 Part A for further details on this protocol) designed to investigate the behavioral signature and mechanisms of neuropsychiatric syndromes such as apathy or disinhibition (Godefroy et al. 2021). For this study, we used data allowing to investigate the behavioral signature and mechanisms of apathy: behavioral measures collected with an ecological approach, clinical measures of apathy (by questionnaires) and MRI data.

\subsection{Behavioral measures in a close-to-real life setting}

\subsubsection{The ECOCAPTURE setting}

The ECOCAPTURE setting reproduced a close-to-real life situation (i.e., being left alone in a "waiting room") with a predetermined script (see Supplementary file 1 Part B for further details on the ECOCAPTURE scenario), whereby participants were firstly left in a freely moving phase called "free phase" (FP) for 7 minutes. This phase was followed by other phases including a "guided phase" (GP) lasting 10 minutes. In GP, participants were asked by the experimenter to fill out a questionnaire, with very easy questions, but requiring in-depth exploration of the room. Thus, the main difference between the FP and GP was that in the FP, participants had to self-initiate goal-directed behaviors whereas in the GP, the initiation of goal-directed behaviors was facilitated by the external assignment of a goal.

During the whole scenario, participants' behavior was video-recorded and their body acceleration was measured using a body sensor (Move II ${ }^{\circledR}$, Movisens, Karlsruhe, Germany) worn on the hip. Figure 2 summarizes how behavioral metrics were measured for both FP and 
GP (see Supplementary file 1 Part $\mathrm{C}$ for further details on behavior coding and extraction of metrics). These behavioral measures extracted from the ECOCAPTURE ecological setting are assumed to be related to apathy defined from a behavioral point of view as a reduction of GDB.

\subsubsection{Activity time and walking metrics}

The activity time ratio (Activity_time_ratio) is a metric quantifying the time spent in goaldirected activity in FP and GP. This is the ratio of time spent in sustained goal-directed actions, visibly organized towards a coherent purpose (e.g., reading a magazine, playing a game). Walking characteristics were assumed to quantify the "goal-directedness" of movements in the room in FP and GP. While exploring the room to select goal-directed actions in FP or searching for the items required to fill the questionnaire in GP, we suggest that three characteristics of the walking episodes can give an indication of how efficient and goal-directed the walk was. These include the number of occurrences of walking episodes (WEp_Occurrences), their mean acceleration (WEp_Acceleration) and their mean duration (WEP_Duration). In the specific ecological situation created for the ECOCAPTURE protocol, we assumed that a reduction of goal-directed behaviors would be manifested in a combination of a decreased time spent in goal-directed actions and a tendency to wander in the room (with frequent, long-lasting walking episodes of low acceleration) without being able to focus on any specific activity. 

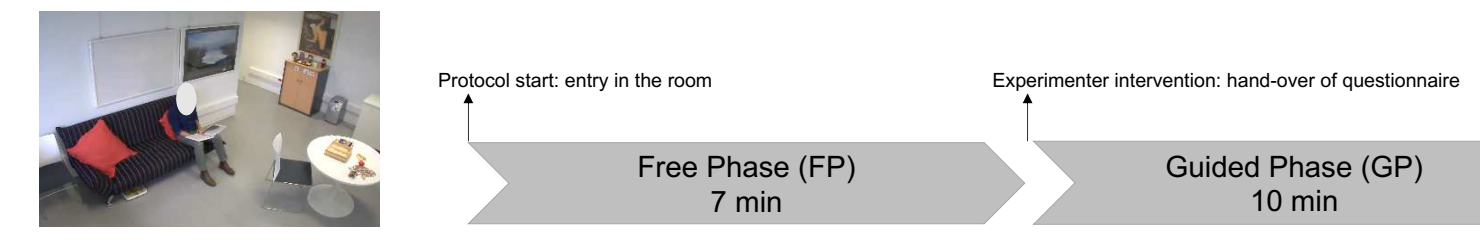

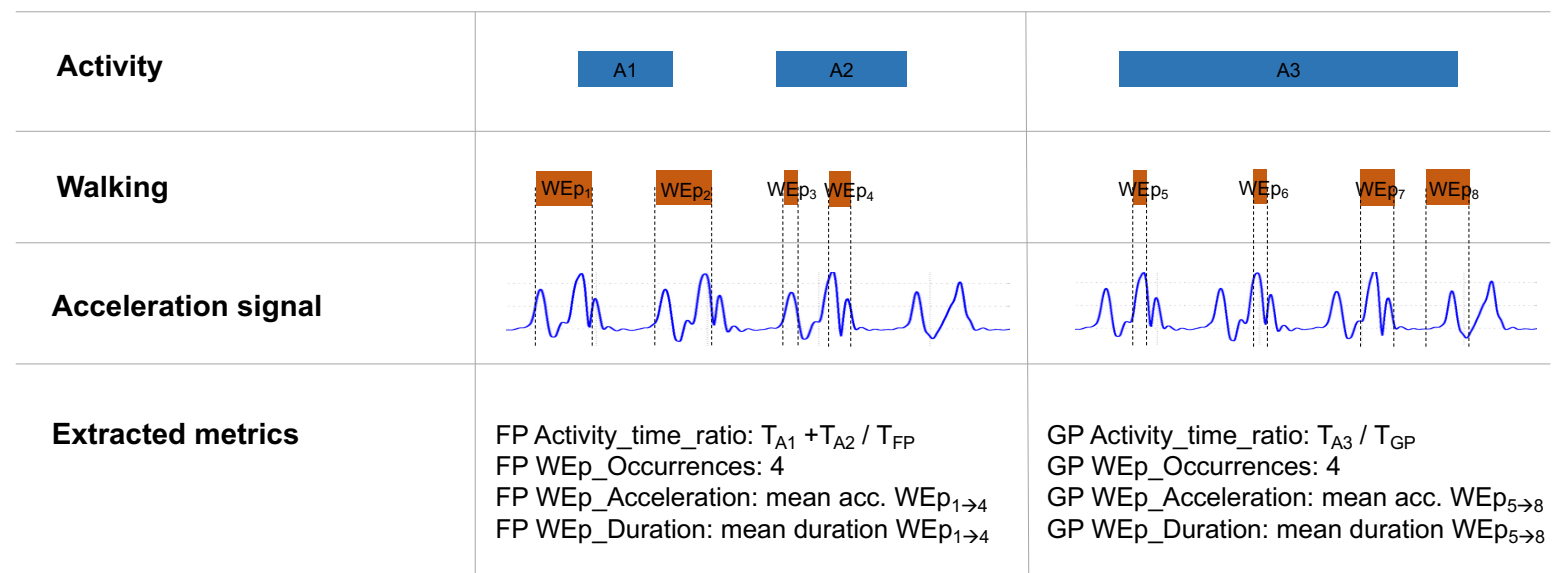

Fig. 1 Summarised methodology of extraction of behavioural metrics. GDB: GoalDirected Behaviours; WEp: Walking Episode; $\mathrm{T}_{\mathrm{A} 1}$ : duration of event A1.; mean acc. WEp $\mathrm{p}_{1 \rightarrow 4}$ : mean acceleration of walking episodes 1 to 4 .

2.3. Neuropsychological tests and measures of apathy by questionnaires

To characterize the bvFTD population in terms of disease severity, we used measures of global mental efficiency and cognitive functions: the Mini-Mental State Evaluation (MMSE) (Folstein et al. 1975), the Mattis Dementia Rating Scale (DRS) (Mattis 1976, 1988) and the Frontal Assessment Battery (FAB) (Dubois et al.). To assess the specific syndrome of apathy, we firstly used the unidimensional Starkstein Apathy Scale (SAS) (Starkstein et al. 1992) (a shortened version of the first scale developed by Marin et al. (Marin et al. 1991)) (14 items; e.g., "Do you have motivation?"). Moreover, we used the Dimensional Apathy Scale (DAS) (Radakovic and Abrahams 2014) which consists of three subscales respectively measuring the Emotional (8 items; e.g., "I become emotional easily when watching something happy or sad on TV"), Initiation (8 items; e.g., "I set goals for myself”) and Executive (8 items; e.g., "I find 
it difficult to keep my mind on things") underlying mechanisms of apathy derived from the theoretical model of apathy subtypes by Levy and Dubois (Levy and Dubois 2006).

\subsection{MRI data acquisition and preprocessing}

MRI data were acquired at the neuroimaging centre (CENIR) of the Paris Brain Institute with a 3-Tesla Siemens Prisma whole-body scanner with a 12-channel head coil. Anatomical data were T1-weighted images acquired using a magnetization prepared rapid acquisition gradient echo pulse sequence (MPRAGE). Resting-state functional data based on the blood oxygenation level-dependent (BOLD) signal were acquired using a T2*-weighted echo-planar image (EPI) pulse sequence with the following parameters: $\mathrm{TR}=2050 \mathrm{~ms}$, $\mathrm{TE}=25 \mathrm{~ms}$, flip angle $=80^{\circ}$, acquisition matrix $=68 \times 68, \mathrm{FOV}=204 \mathrm{~mm}$. Oblique axial slices of the brain were acquired at 436 time points with a voxel resolution of $3 \mathrm{~mm}$ isotropic. Participants were asked to lie with their eyes closed (without falling asleep) during the resting-state acquisition run. MRI data of a total of 34 participants $(18$ bvFTD and $16 \mathrm{HC})$ is reported here since the resting-state functional data of two bvFTD patients were either missing or unusable (because of a concomitant vascular disorder potentially impacting the BOLD signal).

MRI data were preprocessed with the default preprocessing pipeline of the CONN toolbox, an open-source Matlab/SPM-based cross-platform software for the analysis of functional connectivity MRI (Whitfield-Gabrieli and Nieto-Castanon 2012). First, functional data were realigned using SPM12 realign \& unwarp procedure, where all scans are coregistered and resampled to a reference image (first scan of the first session) using b-spline interpolation. Temporal misalignment between different slices of the functional data was corrected using SPM12 slice-timing correction procedure, where the functional data is timeshifted and resampled using sinc-interpolation to match the time in the middle of each acquisition time. Then potential outlier scans were identified from the observed global BOLD 
signal and the amount of subject-motion in the scanner. Functional and anatomical data were normalized into standard MNI space and segmented into grey matter, white matter, and CSF tissue classes using SPM12 unified segmentation and normalization procedure. Last, functional data were smoothed using spatial convolution with a Gaussian kernel of $8 \mathrm{~mm}$ full width half maximum, in order to increase BOLD signal-to-noise ratio and reduce the influence of residual variability in functional and gyral anatomy across subjects.

\subsection{Analyses of behavioral data}

The behavioral analyses detailed in the following subsections aimed to: $1 /$ test the behavioral metrics extracted from the ECOCAPTURE setting as potential behavioral markers of apathy; 2/ disentangle a dimension characterizing the global reduction of goal-directed activity and another dimension corresponding to the specific deficit of self-initiation of goal-directed activity. All these analyses were performed using R Studio (Version 1.2.1335).

\subsubsection{Test of behavioral metrics as potential apathy measures}

We firstly tested the hypothesis that bvFTD patients, characterized by high levels of apathy, would behave differently from $\mathrm{HC}$ on the behavioral metrics extracted from the ECOCAPTURE setting. For this purpose, we used a mixed ANOVA design and explored the main and interaction effects of group (between-subjects factor; bvFTD vs HC) and phase (within-subjects factor; FP vs GP) on the raw activity time and walking metrics (extracted in FP and GP). For all these ANOVA analyses, the normality of residuals (in each group x phase condition) was systematically checked and measures identified as extreme outliers using boxplot methods were removed to better respect the hypothesis of normality. Whenever this hypothesis was not totally satisfied, we further explored the effects of group and phase separately using non-parametric Wilcoxon tests. Besides, we investigated the Spearman's 
rank correlations (across bvFTD patients and $\mathrm{HC}$ ) between apathy measured by the SAS and the means calculated on the total of FP and GP for activity and walking metrics (e.g., $\operatorname{Mean}_{(F P+G P)}$ Activity_time_ratio).

\subsubsection{Dimensional analysis of behavioral metrics and apathy measures by questionnaire}

For this analysis, we used two types of measures: 1/ for each of the four behavioral metrics, the calculated means on the total of FP and GP (e.g., Mean $(F P+G P)$ Activity_time_ratio) assumed to represent the global reduction of goal-directed behaviors and the calculated differences between FP and GP (e.g., Delta $(F P-G P)$ Activity_time_ratio) as potential indicators of the specific (reversible) deficit of self-initiation; 2/ the different measures of apathy and apathy subtypes by questionnaires (SAS and DAS subscales). To disentangle the hypothetical dimensions of global reduction of goal-directed activity and specific self-initiation deficit, we used an Exploratory Factor Analysis (EFA) across bvFTD patients and HC, with a promax oblique rotation and a weighted least square estimator. EFA enables the identification of common dimensions underlying the relationships between observed variables. Prior to the extraction of factors, we used the Kaiser-Meyer-Olkin (KMO) Measure of Sampling Adequacy and Bartlett's Test of Sphericity to assess the suitability of data for EFA. The recommended minimum value for the KMO index is 0.6 and the Bartlett's Sphericity Test should be significant at $p<.05$ (Tabachnick and Fidell 2007; Hair et al. 2010). We used the scree plot of eigenvalues (Cattell 1966) to investigate the number of factors to extract. To reach a reliable factor solution, we iteratively removed items considered as unrelated to the extracted factors (i.e., with a loading $<.30$ on all factors) (Raubenheimer 2004) and we assessed the internal consistency of the extracted components using Cronbach's alpha (with a cut-off of .60). Individual z-scores on the two extracted factors were automatically calculated using individual data on all measures included in the EFA. 
2.6. Analyses of resting-state functional data

We explored the relationship between the extracted apathy-related dimensions and: $1 /$ the whole-brain fractional amplitude of low-frequency fluctuation (fALFF) as an index of local functional activity; 2/ the seed-based correlation (SBC) maps as indicators of the distant functional connectivity between several network hubs and the rest of the brain. All these analyses were performed using the CONN toolbox (version CONN20.b).

\subsubsection{Fractional amplitude of low-frequency fluctuation}

Given the importance of low-frequency fluctuations in determining resting-state activity, the analysis of low-frequency signal power has emerged as a fruitful approach to characterizing the local health of resting-state networks (Zou et al. 2008). The fALFF was calculated for each participant as the voxelwise ratio between low-frequency power (i.e., $0.01-0.1 \mathrm{~Hz}$ ) and the broader-frequency spectrum of resting-state activity (i.e., 0-0.25 Hz).

\subsubsection{Seed-based correlation maps}

SBC maps represent the level of functional connectivity between a seed and every voxel in the brain. SBC maps were generated for each participant by calculating the Fishertransformed bivariate correlation coefficients between each individual voxel BOLD timeseries and the BOLD timeseries averaged over each of seven seed regions of interest. These seeds corresponded to three major hubs of the SN: anterior cingulate cortex (ACC), left and right anterior insula (AI), and four major hubs of the DMN: medial prefrontal cortex (MPFC), posterior cingulate cortex (PCC), left and right lateral parietal cortices (LPC). These selected SN and DMN hubs were assumed to play a key role in the physiopathology of bvFTD (Zhou et al. 2010; Zhou and Seeley 2014) and in the generation of goal-directed 
behaviors (Raichle et al. 2001). To this end, the seeds provided in CONN were used, which were obtained by independent component analysis of 497 normal subjects from the Human Connectome Project dataset. The MNI coordinates of the centers of mass of the seven selected seeds are reported hereafter: ACC $(0,22,35)$, left AI $(-44,13,1)$, right AI $(47,14,0)$, MPFC (1, 55, -3), PCC (1, -61, 38), left LPC $(-39,-77,33)$ and right LPC $(47,-67,29)$.

\subsubsection{Group-level analyses}

The whole-brain fALFF and SBC maps of the seven selected seeds were generated for 18 bvFTD and $16 \mathrm{HC}$. Then we tested the respective effects of the two apathy-related dimensions (extracted by EFA) on fALFF and on each SBC map, using two separate multiple regression analyses (one for each dimension) within the general linear model framework. Age and sex were included as nuisance covariates in all the tested models. For cluster level inference, we used a standard criterion for thresholding voxel-based functional activation/connectivity spatial parametric maps while appropriately controlling the familywise error rate. We used Random Field Theory with a combination of an uncorrected $\mathrm{p}<0.001$ as voxel-level threshold to initially define clusters of interest, and an FDR-corrected $\mathrm{p}<0.05$ (i.e., corrected for false discovery rate) as cluster-level threshold to select among the resulting clusters those deemed significant.

\section{RESULTS}

\subsection{Characteristics of participants}

The demographic and neuropsychological characteristics of bvFTD patients and HC are described in Table 1. We found no significant difference between bvFTD and HC for age, sex and education level. BvFTD patients presented significantly lower MMSE, DRS and FAB scores as compared to $\mathrm{HC}(P<.001)$. Fifteen out of 20 bvFTD patients $(75 \%)$ were above the 
Starkstein Apathy Scale pathological cut-off (i.e., $S A S \geq 14$ ), while none of the HC were. As expected, $S A S(P<.001)$ was significantly higher in bvFTD than in HC. 
Table 1 - Demographical, cognitive and apathy characteristics of bvFTD patients and $\mathrm{HC}^{1}$

\begin{tabular}{llll}
\hline & bvFTD & HC & bvFTD vs HC \\
\hline \% Women & $35 \%$ & $62.5 \%$ & $\mathrm{p}=.19^{\mathrm{a}}$ \\
Age & $65.8(8.8)$ & $62.9(7.6)$ & $\mathrm{p}=.29^{\mathrm{b}}$ \\
Years since 1 ${ }^{\text {st }}$ symptoms & $4.4(2.3)$ & - & - \\
Education level & $6.4(2.0)$ & $7.3(1.1)$ & $\mathrm{p}=.34^{\mathrm{c}}$ \\
\hline MMSE (/30) & $24.1(2.8)$ & $29.4(0.8)$ & $\mathrm{p}<.001^{\mathrm{c}}$ \\
DRS (/144) & $119.5(9.3)$ & $142.3(1.3)$ & $\mathrm{p}<.001^{\mathrm{c}}$ \\
FAB (/18) & $12.5(3.4)$ & $17.3(0.9)$ & $\mathrm{p}<.001^{\mathrm{c}}$ \\
\hline SAS (/42) & $15.4(4.8)$ & $6.3(2.8)$ & $\mathrm{p}<.001^{\mathrm{b}}$ \\
DAS (/72) & $30.4(10.5)$ & $19.6(8.6)$ & $\mathrm{p}<.01^{\mathrm{b}}$ \\
DAS-Emotional (/24) & $10.9(3.9)$ & $8.3(3.4)$ & $\mathrm{p}<.05^{\mathrm{b}}$ \\
DAS-Initiation (/24) & $9.9(5.9)$ & $7.0(4.0)$ & $\mathrm{p}=.09^{\mathrm{b}}$ \\
DAS-Executive (/24) & $9.7(5.0)$ & $4.3(3.7)$ & $\mathrm{p}<.01^{\mathrm{c}}$
\end{tabular}

${ }^{1}$ Data are given as Mean (SD). BvFTD: $\mathrm{N}=20$ / HC: $\mathrm{N}=16$;

${ }^{\mathrm{a}}$ Chi-square test; ${ }^{\mathrm{b}}$ Student t-test; ${ }^{\mathrm{c}}$ Wilcoxon test. BvFTD: behavioural variant of Frontotemporal Dementia; HC:

Healthy Control; MMSE: Mini-Mental State Examination; DRS: Dementia Rating Scale; FAB: Frontal Assessment Battery; SAS: Starkstein Apathy Scale; DAS: Dimensional Apathy Scale; n.s.: non-significant. 


\subsection{Investigation of behavioral metrics as objective markers of apathy}

\subsubsection{Effects of group and phase on behavioral metrics}

We found significant main effects of group $(P<.05)$ and phase $(P<.001)$ on the total activity time ratio but no interaction effect (see Fig. 2.A). Both bvFTD patients and HC showed increased activity time in GP compared to FP but overall, bvFTD patients showed less activity time than HC. As the hypothesis of normality of residuals was not fully satisfied, we confirmed this result by investigating separately the effects of group and phase on activity time using Wilcoxon tests. There was only a significant main effect of phase $(P<.01)$ on walking episode occurrences, the initiation of walking being overall increased in GP compared to FP (see Fig. 2.B). This was also confirmed when investigating separately the effects of group and phase on walking episode occurrences using Wilcoxon tests. However, when exploring the effect of phase within each group (using Wilcoxon tests), we found that HC presented a significant increase in walking episode occurrences in GP compared to FP ( $P$ $<.001)$ but bvFTD patients did not $(P=.31)$. Regarding the acceleration characteristics of walking episodes (in Fig. 2.C), we found significant main effects of group $(P<.05)$ and phase $(P<.05)$. The mean acceleration of walking was higher in GP compared to FP in both groups and was lower in bvFTD patients compared to HC in both phases. Finally, we observed a significant impact of group $(P<.05)$ on walking episode mean duration, with walking episodes lasting longer in bvFTD compared to HC in both phases (see Fig. 2.D). Altogether, these findings showed that bvFTD patients behaved differently from HC on the extracted activity time and walking metrics. 


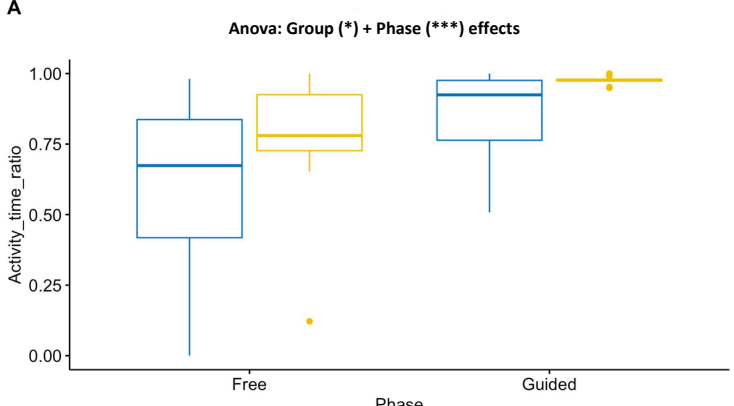

C

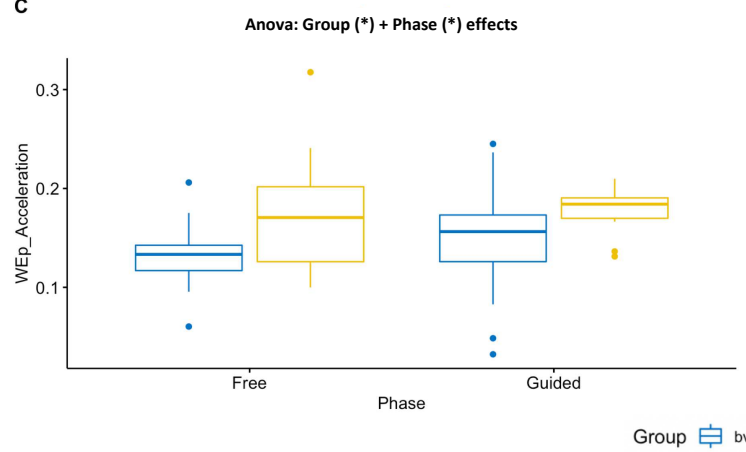

Group 追 bvFTD $\risingdotseq$ Contro
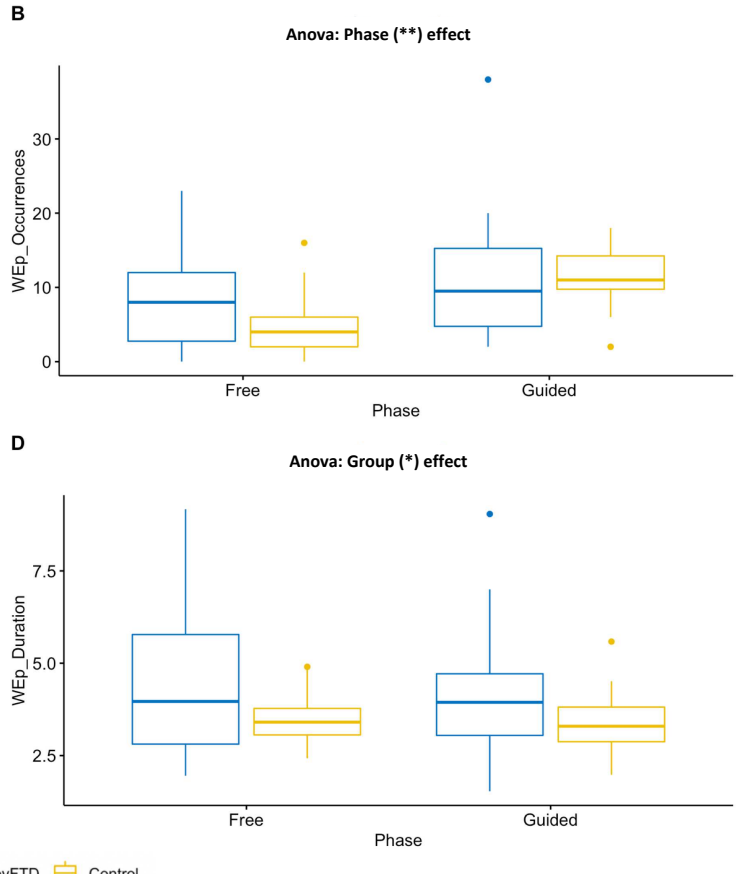

Fig. 2 Effects of group and phase on four behavioural metrics quantifying GDB.

(A) Activity time ratio. (B) Occurrences of walking episodes. (C) Mean acceleration of walking episodes. (D) Mean duration of walking episodes. BvFTD patients: $\mathrm{N}=20$; HC: $\mathrm{N}=16$. Levels of significance of indicated main effects: $(*) P<.05 ;(* *) P<.01 ;(* * *) P<$ .001 .

\subsubsection{Correlations between mean behavioral metrics and $S A S$}

When investigating spearman correlations between SAS and the means of behavioral metrics (on the total of FP and GP), we found that all the behavioral metrics evolved with SAS in the expected way: when SAS increased, activity time and walking acceleration decreased while walking episode occurrences and mean duration increased. We found that correlations with SAS were significant for the calculated metrics of $\operatorname{Mean}_{(F P+G P)}$ Activity_time_ratio $(r=-.37$; $P<.05)$ and $\operatorname{Mean}_{(F P+G P)}$ WEP_Acceleration $(r=-.43 ; P<.05)$, and close-to-significant for $\operatorname{Mean}_{(F P+G P)}$ WEp_Duration $(r=.31 ; P=.07)$. As for walking episode occurrences, their 
mean on the total of FP and GP was not significantly correlated to SAS $(r=.21 ; P=.23)$. However further exploration led to observe that walking episode occurrences extracted exclusively in the FP were significantly correlated to SAS $(r=.43 ; P<.01)$.

Therefore, between-group comparisons and correlation analyses are in agreement with the following assumption: in the context of ECOCAPTURE setting, a low time spent in goaldirected activity and walking episodes of low acceleration, high duration, and of high frequency in the FP, are objective behavioral markers of a reduction of goal-directed behaviors. Activity time and walking features can thus contribute to the assessment of apathy.

\subsection{Disentangling of two dimensions of apathy}

For the EFA applied to the calculated behavioral metrics (means and deltas) and apathy measures by questionnaire, we firstly calculated the overall KMO index. As it was initially found to be inferior to .60 , we explored the KMO value for each item included in the EFA. We had to remove the item with the lowest KMO (i.e., Delta ${ }_{(F P-G P)}$ Activity_time_ratio) from the analysis to reach an overall $\mathrm{KMO}>.60$ for a possible dimension reduction. Bartlett's Test of Sphericity was significant $(P<.05)$, which further confirmed the suitability of data for EFA. Besides, the scree plot analysis confirmed that a two-factor solution was the best. The final factor solution reached with the EFA is shown in Table 1. We obtained a two-factor structure accounting for $36 \%$ of the total variance. We had to remove one of the included items: Delta $(F P-G P)$ WEP_Occurrences (which had a loading $<.30$ on the two extracted factors) in order to get a more reliable solution (i.e., so that the internal consistencies of items loading on F1 and on F2 were both $>.60$ ). Besides, between-group comparisons on extracted scores confirmed that both F1 $(P<.001)$ and F2 $(P<.05)$ dimensions were significantly higher in bvFTD compared to HC. 
All the means of extracted behavioral metrics loaded on $F 1$ as well as the SAS and DASExecutive scores. Therefore $F 1$ was assumed to correspond to the expected dimension of global reduction of goal-directed activity. According to the signs of observed loadings, F1 was a pattern characterized by: low time spent in goal-directed activities, walking episodes of low acceleration, high frequency and high duration, combined with high self-reported lack of motivation for goal-directed activity and high self-reported inability to manage goals.

All the deltas of behavioral metrics loaded on F2 along with DAS-Initiation and DASEmotional scores. Thus F2 was supposed to correspond to the expected dimension assessing the specific deficit of self-initiation of goal-directed activity. According to the signs of observed loadings, F2 was a pattern characterized by: higher mean acceleration and lower mean duration of walking episodes in GP as compared to FP combined with high selfreported lessened initiation of thoughts/behaviors and high self-reported diminished integration and expression of emotions. 
Table 2 - Results of the Exploratory Factor Analysis ${ }^{1}$

Observed variables

Factor loadings

\begin{tabular}{|c|c|c|}
\hline & F1 & F2 \\
\hline SAS score & 0.85 & 0.27 \\
\hline DAS-Executive score & 0.66 & 0.11 \\
\hline $\operatorname{Mean}_{(\mathrm{FP}+\mathrm{GP})}$ Activity_time_ratio & -0.48 & 0.21 \\
\hline $\operatorname{Mean}_{(\mathrm{FP}+\mathrm{GP})}$ WEp_Acceleration & -0.36 & 0.02 \\
\hline $\operatorname{Mean}_{(\mathrm{FP}+\mathrm{GP})}$ WEp_Duration & 0.34 & 0.12 \\
\hline Mean $_{(\mathrm{FP}+\mathrm{GP})}$ WEp_Occurrences & 0.33 & -0.05 \\
\hline DAS-Initiation score & 0.21 & 0.67 \\
\hline Delta(FP-GP) WEp_Duration & -0.12 & 0.63 \\
\hline DAS-Emotional score & 0.14 & 0.60 \\
\hline $\operatorname{Delta}_{\text {(FP-GP) }}$ WEp_Acceleration & 0.12 & -0.53 \\
\hline
\end{tabular}

${ }^{1}$ Values indicate the factor loadings of the EFA. Coefficients in bold represent the highest loading (among the two factors) for each item. The calculation of individual scores on F1 and F2 takes into account all the factor loadings onto $\mathrm{F} 1$ and $\mathrm{F} 2$ respectively. $\mathrm{N}=36$ (bvFTD: $\mathrm{N}=20 / \mathrm{HC}: \mathrm{N}=16)$. Mean $(\mathrm{FP}+\mathrm{GP})$ : mean calculated on the total of FP and GP ((FP+GP)/2); Delta(FP-GP): delta calculated as the difference between FP and GP (FP-GP); Activity_time_ratio: ratio of time spent in goal-directed activity; WEp_Occurrences: occurrences of walking episodes; WEp_Acceleration: mean acceleration of walking episodes; WEp_Duration: mean duration of walking episodes; SAS: apathy defined as a global lack of motivation (assessed as a unidimensional construct); DASEmotional: diminished integration, processing and expression of emotions; DAS-Initiation: lessened initiation of thoughts and actions; DAS-Executive: inability to manage goals and cognitively strategize to execute a plan of actions. 


\subsection{Resting state functional connectivity measures and apathy dimensions}

\subsection{1. fALFF and apathy dimensions}

Figure 3 and Table 3 show the results of group-level analysis regarding the effect of F1 dimension (i.e., global reduction of goal-directed activity) on fALFF index. We did not isolate any significant cluster when exploring the effect of F2 (i.e., specific deficit of self-initiation) on fALFF. Across participants, F1 dimension was negatively related to low-frequency fluctuations in several regions of the prefrontal cortex (PFC): the dorsomedial prefrontal cortex (DMPFC), the left and right dorsolateral prefrontal cortex (DLPFC) and the right orbitofrontal cortex (OFC).

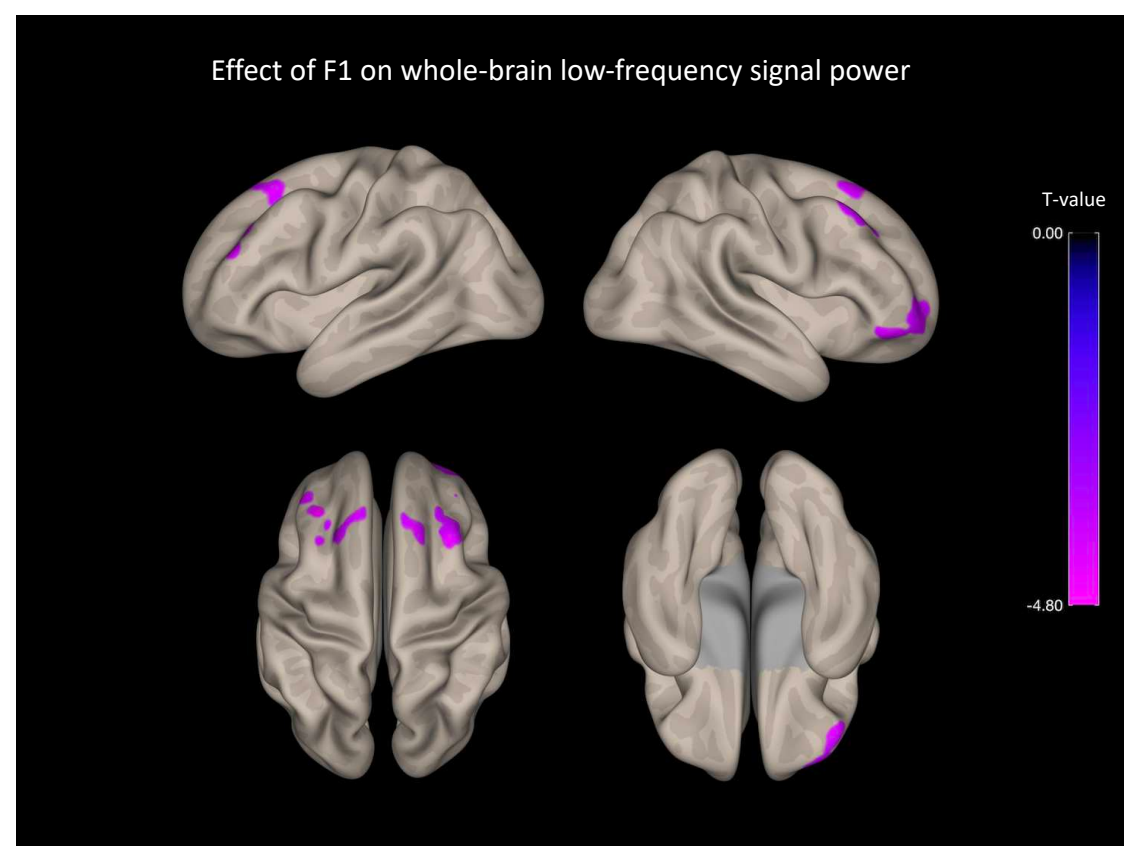

Fig. 3 Negative association between F1 and fALFF index of signal power in several PFC regions. $\mathrm{N}=34$ (bvFTD: $\mathrm{N}=18$ / $\mathrm{HC}$ : $\mathrm{N}=16$ ). Effects are corrected for age and sex, and for family-wise error at the level of individual clusters at $P<.05$. F1: global reduction of goaldirected activity. 
Table 3 - List of significant clusters for group-level analyses on voxelwise low-frequency signal power ${ }^{1}$

\begin{tabular}{llllllll}
\hline Region & L/R & Coordinates & & $\begin{array}{l}\text { Cluster } \\
\text { size }\end{array}$ & $\begin{array}{l}\boldsymbol{p} \text { - } \\
\text { FDR }\end{array}$ & T-score \\
\hline & & $\mathbf{x}$ & $\mathbf{y}$ & $\mathbf{z}$ & & & \\
\hline Effect of F1 on whole brain fALFF & & & & & & & \\
Middle frontal gyrus & $\mathrm{R}$ & 32 & 22 & 58 & 350 & $<.001$ & -5.92 \\
Frontal pole & $\mathrm{R}$ & 46 & 46 & -20 & 125 & .02 & -5.25 \\
Frontal pole & $\mathrm{R}$ & 30 & 58 & -4 & 124 & .02 & -4.70 \\
Middle frontal gyrus & $\mathrm{L}$ & -38 & 34 & 38 & 120 & .02 & -5.32 \\
Superior frontal gyrus & $\mathrm{L}$ & -24 & 26 & 54 & 101 & .04 & -5.28
\end{tabular}

${ }^{1}$ Cluster threshold: $p$-FDR $<.05$; voxel threshold: $p$-uncorrected $<.001 . p$-FDR: FDR-corrected $p$-value on cluster size; L/R: left or right hemisphere; fALFF: fractional amplitude of low-frequency fluctuation. N.B.: no significant cluster was identified when investigating the effect of F2 on whole brain fALFF. 


\subsubsection{Seed-based connectivity and apathy dimensions}

Figure 4 and Table 4 show the results of group-level analysis regarding the separate effects of F1 and F2 dimensions on the functional connectivity of three seeds of the SN and four seeds of the DMN. We found that, across participants, F1 (assessing the global reduction of goaldirected activity) was associated with a decreased connectivity between the three SN hubs (i.e., from ACC seed to left/right frontoinsular regions and from left/right AI seeds to ACC), a decreased connectivity between the ACC and the ventral striatum, and a decreased connectivity between the ACC and the dorsomedial frontal cortex (i.e., supplementary motor area - SMA). Although not shown on Figure 4, F1 was also related to increased connectivity between left / right AI and the cerebellum (medial part). F1 was not related to the connectivity of DMN hubs. On the opposite, F2 (assessing the specific deficit of self-initiation) was linked with an increased connectivity of two DMN hubs (left and right LPC) and was not associated with the connectivity of SN hubs. Indeed, F2 was found to increase with the connectivity between bilateral LPC and medial frontal/parietal cortex. In particular, F2 was associated with an increased connectivity between the right LPC and the medial part of superior frontal gyrus, pre- and postcentral gyri (including SMA). Of note, F2 was also related to decreased connectivity between left LPC and a brain stem region. 


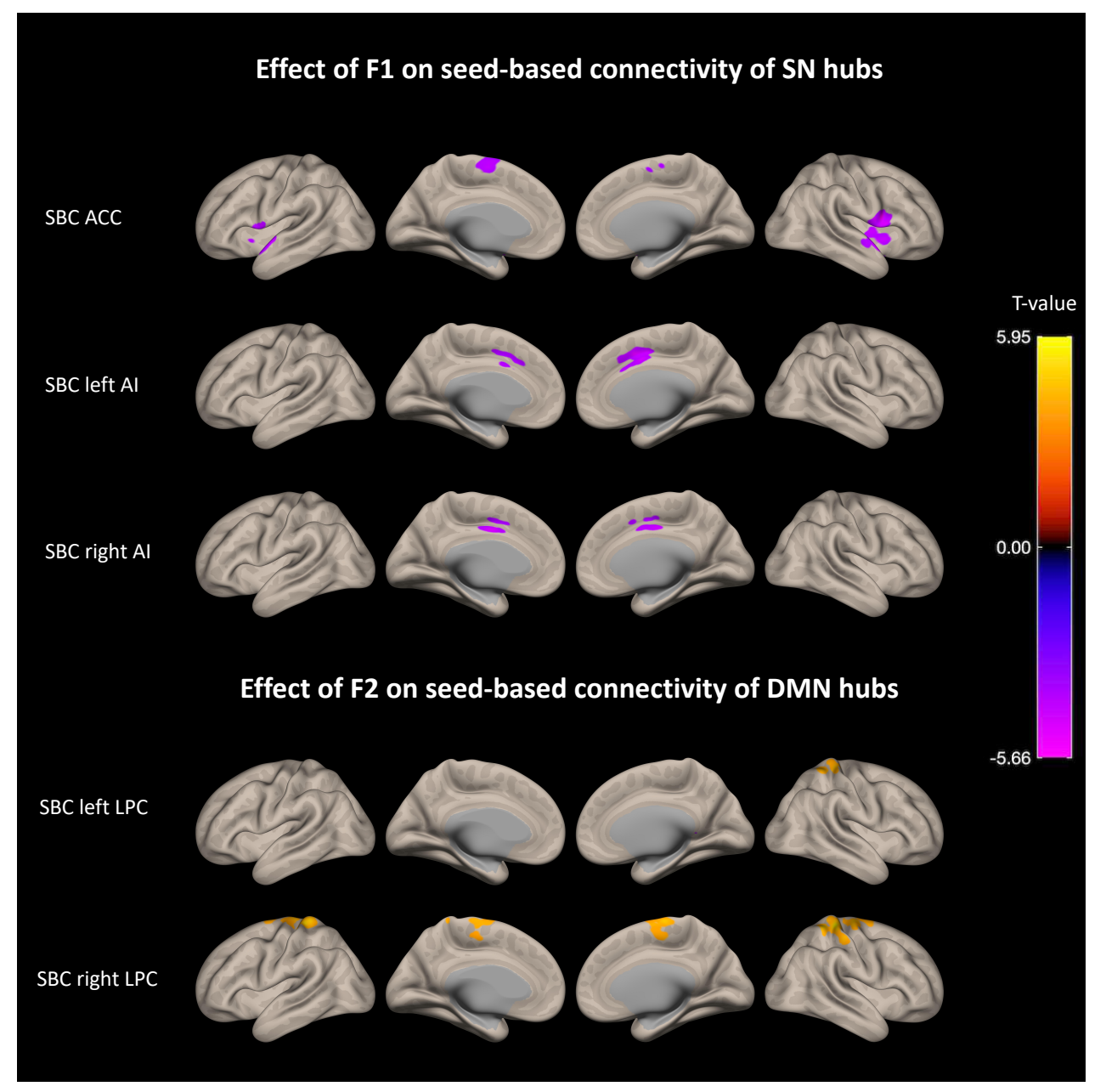

Fig. 4 Associations found between F1/F2 and SN/DMN hubs connectivity. We observed a negative association between F1 and the seed-based connectivity of SN hubs and on the opposite, a positive association between F2 and the seed-based connectivity of two DMN hubs. $\mathrm{N}=34$ (bvFTD: $\mathrm{N}=18$ / $\mathrm{HC}: \mathrm{N}=16$ ). Effects are corrected for age and sex, and for family-wise error at the level of individual clusters at $P<.05$. F1: global reduction of goaldirected activity; F2: specific deficit of self-initiation. 
Table 4 - List of significant clusters for group-level analyses on seed-based connectivity maps $^{1}$

\begin{tabular}{|c|c|c|c|c|c|c|c|}
\hline \multirow[t]{2}{*}{ Region } & \multirow[t]{2}{*}{$\mathbf{L} / \mathbf{R}$} & \multicolumn{3}{|c|}{ Coordinates } & \multirow{2}{*}{$\begin{array}{l}\text { Cluster } \\
\text { size }\end{array}$} & \multirow{2}{*}{$\begin{array}{l}p- \\
\text { FDR }\end{array}$} & \multirow[t]{2}{*}{ T-score } \\
\hline & & $\mathbf{x}$ & $\mathbf{y}$ & $\mathbf{z}$ & & & \\
\hline \multicolumn{8}{|c|}{ Effect of F1 on ACC seed-based connectivity } \\
\hline Precentral gyrus & $\mathrm{R}$ & 52 & 4 & 14 & 967 & $<.001$ & -5.51 \\
\hline Frontal orbital cortex & $\mathrm{R}$ & 26 & 6 & -16 & 425 & .001 & -6.06 \\
\hline Superior temporal gyrus & $\mathrm{L}$ & -62 & 0 & 2 & 410 & .001 & -5.12 \\
\hline Cerebellum & $\mathrm{R}$ & 42 & -48 & -32 & 196 & .03 & -6.03 \\
\hline Accumbens & $\mathrm{L}$ & -14 & 14 & -8 & 184 & .03 & -4.83 \\
\hline Superior frontal gyrus & $\mathrm{L}$ & -6 & 2 & 74 & 172 & .04 & -4.38 \\
\hline \multicolumn{8}{|c|}{ Effect of F1 on left AI seed-based connectivity } \\
\hline Anterior cingulate cortex & $\mathrm{R}$ & 6 & 6 & 46 & 528 & $<.001$ & -4.98 \\
\hline Cerebellum & $\mathrm{R}$ & 16 & -84 & -44 & 278 & .008 & 5.35 \\
\hline Cerebellum & $\mathrm{L}$ & -16 & -84 & -42 & 271 & .008 & 5.88 \\
\hline \multicolumn{8}{|c|}{ Effect of F1 on right AI seed-based connectivity } \\
\hline Cerebellum & $\mathrm{R}$ & 10 & 80 & -40 & 548 & $<.001$ & 6.57 \\
\hline Anterior cingulate cortex & $\mathrm{L}$ & -2 & -2 & 40 & 279 & .008 & -4.85 \\
\hline \multicolumn{8}{|c|}{ Effect of F2 on right LPC seed-based connectivity } \\
\hline Post-central gyrus & $\mathrm{R}$ & 24 & -30 & 70 & 1322 & $<.001$ & 6.83 \\
\hline Post-central gyrus & $\mathrm{L}$ & -22 & -32 & 68 & 424 & $<.001$ & 5.67 \\
\hline \multicolumn{8}{|c|}{ Effect of F2 on left LPC seed-based connectivity } \\
\hline Post-central gyrus & $\mathrm{R}$ & 22 & -32 & 68 & 408 & .001 & 5.42 \\
\hline Brain Stem & $\mathrm{L}$ & -2 & -32 & -4 & 348 & .001 & -7.66 \\
\hline
\end{tabular}


${ }^{1}$ Cluster threshold: $p$-FDR $<.05$; voxel threshold: $p$-uncorrected $<.001 . p$-FDR: FDR-corrected $p$-value on cluster size; L/R: left or right hemisphere; ACC: anterior cingulate cortex; AI: anterior insula; LPC: lateral parietal cortex.

\section{DISCUSSION}

The present investigation on apathy in bvFTD, based on multi-modal assessments including an ecological approach of behavior tracking, allowed us: (i) to identify new behavioral measures contributing to the assessment of apathy; (ii) to disentangle two dimensions describing the global severity of apathy and the specific contribution of a self-initiation deficit; (iii) to evidence that global apathy severity and self-initiation deficit were associated with two distinct patterns of modified functional connectivity.

\subsubsection{New behavioral markers of apathy}

We found that bvFTD patients (known to present high levels of apathy) spent less time in goal-directed activities and presented less "goal-directed" walking episodes (of lower acceleration and higher duration) than $\mathrm{HC}$ did. Both bvFTD patients and $\mathrm{HC}$ similarly increased their time spent in goal-directed activity and the acceleration of walking in the GP compared to the FP. This last result suggested that like HC, bvFTD patients could benefit from external guidance to quantitatively increase goal-directed behaviors. However, no group-phase interaction was evidenced and the discrepancy between bvFTD and HC was maintained in GP, which indicates that hetero-guidance cannot totally compensate for the deficit of goal-directed behaviors in bvFTD.

As confirmed by correlation analyses, using an ecological approach allowed us to put forward novel behavioural markers of apathy in bvFTD patients, in particular the acceleration while walking which was significantly and inversely correlated to SAS apathy score. One 
study (Groeneweg-Koolhoven et al. 2015) has already suggested a relationship between apathy and walking speed but it was not based on an ecological approach. They investigated the correlates of apathy (assessed by Marin's Apathy Evaluation Scale) in non-demented older individuals, either depressed or non-depressed. In depressed older individuals, walking speed (determined by measuring the time needed to complete a six-meter walk) was found to be slower in those with apathy compared to those without apathy. Within the domain of ecological approaches, two studies of ambulatory actigraphy (i.e., continuous acceleration measures for several days thanks to a wrist actigraph) in patients with Alzheimer's disease showed that the patients' apathy scores on neuropsychiatric assessment scales correlated negatively with the mean motor activity (i.e., the mean number of activity counts per minute extracted from the recorded acceleration signal) (David et al. 2010, 2012). Our findings emphasize this relationship further: indeed, we show that not only is the long-term mean motor activity (derived from the acceleration signal) related to apathy but also the mean acceleration exclusively while walking in a room on a short-term period. This result suggests that the goals directing participants' actions may unconsciously impact the way their body moves.

\subsubsection{Two dimensions of apathy}

Calculated means and deltas for the activity time and walking metrics, along with measures of apathy by clinical scales, contributed to extract two dimensions: a first dimension (F1, related to the means of behavioral metrics) assumed to characterize the global reduction of goaldirected behaviors and a second dimension (F2, related to the deltas of behavioral metrics) supposed to represent the specific deficit of self-initiation.

The first extracted dimension corresponded to a behavioral pattern characterized by a low time spent in goal-directed activity and a general tendency to wander in the room. It was 
also related to self-reported apathy (measured by SAS) and to a self-reported difficulty to implement a plan of actions towards goal management (measured by DAS-Executive). This finding indicates that, contrary to a common belief, the reality of apathy syndrome is not limited to standing still and doing absolutely nothing. On the opposite, it can include stereotyped aimless movements, like observed in the most severe cases of apathy in patients with AAD syndrome (Laplane and Dubois 2001), which may precisely prevent from focusing on goal-management.

The second extracted dimension corresponded to a pattern of enhanced 'goaldirectedness' of walking when the initiation of goal-directed behaviors was facilitated by hetero-guidance. Thus, typically, patients with high scores on this second dimension would have specific difficulties in self-initiating goal-directed behaviors but might be able to complete them with external guidance. This dimension was also associated with the selfreported impairment of emotional-affective and auto-activation processing (DAS-Emotional and DAS-Initiation). The emotional-affective aspect of apathy has been shown to be very salient in bvFTD (Kumfor et al. 2018; Radakovic et al. 2021), which may explain that this mechanism contributes to the specific deficit of self-initiation of goal-directed behaviors, along with the difficulty to initiate thoughts and actions. The executive / cognitive mechanism of apathy was not related to the dimension of self-initiation deficit, maybe because it is mostly associated with the capacity to sustain (and not to initiate) goal-directed behaviors.

\subsubsection{Functional connectivity related to the two dimensions of apathy}

The F1 dimension of reduction of goal-directed behaviors (or global apathy) was related to lower low-frequency signal power within several distinct regions of the PFC: the right OFC, the bilateral DLPFC and DMPFC. These regions correspond to the frontal part of the three frontostriatal circuits associated with apathy in the model proposed by Levy and colleagues 
(Levy and Dubois 2006; Levy 2012; Godefroy et al. 2020). In this model, the emotionalaffective (or 'loss of motivation') subtype is assumed to be related to the "orbital-ventralmesial circuit" linking the orbital and ventromedial PFC to the ventral regions of the basal ganglia. Cognitive inertia is attributed to damage to the "dorsolateral circuit" linking the DLPFC to the dorsal regions of the basal ganglia. Finally, the auto-activation deficit is associated with the "anterior cingulate-dorsal mesial circuit" linking the ACC and DMPFC to basal ganglia portions located between the dorsal and ventral regions. This model was partly supported by empirical evidence using structural imaging. Indeed, Massimo and colleagues (Massimo et al. 2015) assessed three components of goal-directed behaviors (i.e., motivation, planning, and initiation) in bvFTD patients using the Philadelphia Apathy Computerized Test (PACT) and they found that poor motivation was related to grey matter atrophy in OFC, planning impairment to atrophy in DLPFC, and poor initiation to atrophy in ACC.

Interestingly, there was no overlap between the brain regions for which local resting state activity was related to global apathy (i.e., OFC, DLPFC and DMPFC) and the brain regions for which distant connectivity was associated with global apathy (i.e., mostly ACC, left/right AI, ventral striatum and SMA). The investigation of local resting state activity in association with apathy evidenced PFC areas of which grey matter atrophy was consistently related to apathy (or apathy subtypes) in previous literature regarding FTD symptoms (Ducharme et al. 2018). On the opposite, the analysis of distant connectivity from several seeds revealed the involvement of regions that were less typically associated with apathy by structural neuroimaging in FTD, like the AI or the SMA (Ducharme et al. 2018), or even regions found to be associated with apathy on isolated occasions like the cerebellum (Shaw et al. 2021) or the brain stem (ventral tegmental area) (Schroeter et al. 2011). Therefore, the global reduction of goal-directed behaviors may arise from the complex combination of: 1/ local changes in connectivity in close association with local grey matter atrophy and 2/ global 
changes in connectivity between more distant regions of the brain that are less directly related to grey matter atrophy.

Through the analysis of the distant functional connectivity between selected hubs of the SN / DMN and the rest of the brain, we identified two distinct patterns of modified connectivity relating to the two dimensions of apathy. A decreased connectivity between SN hubs (AAC and bilateral AI) correlated with global apathy while an increased connectivity of DMN hubs (bilateral LPC) with dorsomedial regions correlated with the specific deficit of self-initiation. These findings were in agreement with the model of connectivity changes in bvFTD suggested by Zhou, Seeley and colleagues (Zhou et al. 2010; Zhou and Seeley 2014). These authors had shown that global clinical severity in bvFTD correlated with loss of right frontoinsular SN connectivity and enhancement of parietal DMN connectivity, suggesting that functional connectivity reductions and enhancements both carried the potential to track the progression of disease-related disorders (Zhou et al. 2010). Besides, two previous studies of MRI resting state functional connectivity in bvFTD also found links between apathy (assessed as a unidimensional construct with the Frontal Behavioral Inventory) and the connectivity within SN (Day et al. 2013; Farb et al. 2013) and within DMN (Farb et al. 2013). An additional study (Zhou et al. 2020) applied a graph theoretical analysis to analyze the topological properties of cerebral blood flow network based on single photon emission tomography in bvFTD patients with and without apathy. They observed that, compared with bvFTD patients without apathy, patients with apathy exhibited additional loss of hubs in regions commonly associated with the SN (including ACC, AI, ventral rostral PFC areas, and subcortical regions), but recruited more hubs in areas belonging to the DMN (including angular gyrus, precuneus and posterior cingulate cortex).

Results suggested that the connectivity of ACC plays a key role in the quantitative production (both initiation and maintenance) of goal-directed behaviours. As predicted by 
Zhou and Seeley's working functional-anatomic model of the SN, the functional connectivity between the ACC and AI (or frontoinsula - FI) was involved in the severity of global apathy syndrome (assessed by F1). According to this model, the ACC node coordinates with the AI, which processes the major ascending input streams regarding the moment-to-moment condition of the body to elaborate 'feeling state' representations. According to the 'feeling state' communicated by the AI, the ACC can mobilize adapted behavioural responses through: 1. the recruitment of executive and task control network resources; 2 . the inhibition of the DMN to keep attention focused on the ongoing task. Previous studies also found a relationship between the connectivity of dorsal ACC and apathy in patients with AD (Tumati et al. 2020), in patients with late-life depression (Yuen et al. 2014) and in patients with Parkinson's disease (Sun et al. 2020). Besides, the functional connectivity between dorsal ACC and SMA had been shown to decrease with apathy as a trait in healthy subjects (Bonnelle et al. 2016), which was replicated in our study in a sample mixing bvFTD patients and healthy controls.

Results also evidenced an important role of increased connectivity between parietal DMN hubs (in particular the right LPC) and dorsomedial regions of motor and pre-motor cortices in the specific ability to self-initiate goal-directed behaviors. The activation of the DMN is generally recognized to be responsible for an introspective state which prevents the allocation of cognitive resources on an extrinsic goal-directed task (Mak et al. 2017). An increased connectivity of the DMN may reduce the capacity to intrinsically deactivate this network and thus disturb the self-initiation of goal-directed behaviors. More specifically, the involvement of LPC is in line with a recent review of literature on the association between LPC dysfunction and apathy across disorders (Tumati et al. 2019). Considering the evidence, the authors proposed that the neural processes in the lateral parietal lobule may contribute to transform internal goals into external actions through a process of embedding intended actions 
in a 'body schema' which facilitates the adequate recruitment of an effector system. An increased connectivity between the LPC and medial motor / pre-motor regions may thus reflect an inadequate recruitment of an effector system by the LPC and constitute a compensatory mechanism for a disturbed embedding of intended actions in a 'body schema'. Tumati and colleagues suggested a revised model of apathy in which impaired internal initiation of behavior mediated by a dysfunction of the lateral parietal lobule may be sufficient (although not necessary) to reduce goal-directed behavior, and would constitute a volitional subtype of apathy.

Finally, results suggested the importance of SMA as an efferent motor system involved in both the global apathy and the specific self-initiation deficit. In bvFTD patients, the global reduction of goal-directed behaviours might be related to the decreased connectivity of ACC with afferent salience nodes (i.e., AI) and efferent motor nodes (i.e., SMA). In some patients with relatively preserved connectivity of ACC, LPC dysfunctions combined with an enhanced connectivity between LPC and SMA might be sufficient to prevent the spontaneous self-initiation of goal-directed behaviors without suppressing the capacity to generate these behaviors with hetero-guidance. This is consistent with the assumption that dorsomedial frontal areas are linked to the initiation component of apathy (Ducharme et al. 2018). In general, the pre-SMA/SMA region, which integrates inputs from the PFC and basal ganglia, is assumed to play a major part in the initiation of voluntary action (Haggard 2008).

\section{Methodological limits}

The relatively small sample size of bvFTD patients is a first limitation of the present study. Small sample size is a common issue in studies investigating the neural bases of apathy in FTD (Ducharme et al. 2018). This is due to the heavy requirements of our protocol (two days 
of experimental protocol with extensive neuropsychological testing) and to our selective inclusion criteria for bvFTD patients (e.g., MMSE score > 20).

The extent of the impact of anosognosia, potentially biasing subjective measures of apathy in bvFTD patients (Levy 2012), is difficult to estimate because we lack reports from the caregiver. However, in this research, subjective bias was compensated for by the addition of objective behavioral measures to form composite dimensions describing apathy more thoroughly.

\section{Conclusions}

This research opens the way for the development of a new evaluation of apathy across patients and healthy individuals. Our study highlights that along with the time spent in activity, walking characteristics under close-to-real-life situations could be very useful in complementing subjective measures of apathy, thus leading to a more thorough and precise quantification of apathy. This assessment approach combining objective and subjective measures could be extended to a broad range of behavioral symptoms and could thus prove useful for a better understanding of the relationship between brain connectivity and behavior.

Our results go beyond the already evidenced associations between different mechanisms of apathy and the atrophy of distinct brain regions of the PFC. Using the lesional model provided by bvFTD patients, we showed that a reduction of goal-directed behaviors was associated with the combination of a decreased local connectivity within the PFC and a decreased distant connectivity of SN hubs (in particular of the ACC node), in accordance with Zhou and Seeley's working model of the SN (Zhou and Seeley 2014). Moreover, the (reversible) selfinitiation deficit was related to an increased connectivity between right LPC and a dorsomedial region including the SMA, which confirmed the key roles of these regions in the specific ability to self-initiate voluntary goal-directed behaviors (Haggard 2008; Tumati et al. 
2019). We suggest that the enhanced connectivity between LPC and SMA could serve as a biomarker of impaired volition. Further investigation into the longitudinal evolution of functional connectivity changes leading to apathy and to self-initiation deficit in bvFTD could provide further insight into the causal relationships between connectivity and the production of goal-directed behaviors.

\section{REFERENCES}

Ang Y-S, Lockwood P, Apps MAJ, et al (2017) Distinct Subtypes of Apathy Revealed by the Apathy Motivation Index. PLOS ONE 12:e0169938.

https://doi.org/10.1371/journal.pone.0169938

Batrancourt BM, Lecouturier K, Ferrand-Verdejo J, et al (2019) Exploration deficits under ecological conditions as a marker of apathy in frontotemporal dementia. Frontiers in neurology 10:941

Bonnelle V, Manohar S, Behrens T, Husain M (2016) Individual differences in premotor brain systems underlie behavioral apathy. Cerebral cortex 26:807-819

Cattell RB (1966) The scree test for the number of factors. Multivariate behavioral research $1: 245-276$

David R, Mulin E, Friedman L, et al (2012) Decreased Daytime Motor Activity Associated With Apathy in Alzheimer Disease: An Actigraphic Study. The American Journal of Geriatric Psychiatry 20:806-814. https://doi.org/10.1097/JGP.0b013e31823038af

David R, Rivet A, Robert PH, et al (2010) Ambulatory actigraphy correlates with apathy in mild Alzheimer's disease. Dementia 9:509-516. https://doi.org/10.1177/1471301210381678

Day GS, Farb NAS, Tang-Wai DF (2013) Salience Network Resting-State Activity:

Prediction of Frontotemporal Dementia Progression. JAMA neurology, 70:1249-1253. 
Dubois B, Slachevsky A, Litvan I, Pillon B A frontal assessment battery at bedside. Neurology 69:680-694. https://doi.org/0.1212/WNL.55.11.1621

Ducharme S, Price BH, Dickerson BC (2018) Apathy: a neurocircuitry model based on frontotemporal dementia. J Neurol Neurosurg Psychiatry 89:389-396.

https://doi.org/10.1136/jnnp-2017-316277

Farb NAS, Grady CL, Strother S, et al (2013) Abnormal network connectivity in frontotemporal dementia: Evidence for prefrontal isolation. Cortex 49:1856-1873. https://doi.org/10.1016/j.cortex.2012.09.008

Folstein MF, Folstein SE, McHugh PR (1975) “Mini-mental state”. A practical method for grading the cognitive state of patients for the clinician. J Psychiatr Res 12:189-198. https://doi.org/10.1016/0022-3956(75)90026-6

Godefroy V, Batrancourt B, Levy R (2020) Apathy: From the Underlying Pathophysiological Mechanisms to Future Assessments and Therapeutic Strategies. In: Della Sala S (ed) Reference Module in Neuroscience and Biobehavioral Psychology. Elsevier. https://doi.org/10.1016/B978-0-12-819641-0.00057-8

Godefroy V, Tanguy D, Bouzigues A, et al (2021) Frontotemporal dementia subtypes based on behavioral inhibition deficits. Alzheimer's \& Dementia: Diagnosis, Assessment \& Disease Monitoring 13:e12178

Groeneweg-Koolhoven I, Comijs HC, Naarding P, et al (2015) Presence and correlates of apathy in non-demented depressed and non-depressed older persons. The European Journal of Psychiatry 29:119-130

Haggard P (2008) Human volition: towards a neuroscience of will. Nature Reviews Neuroscience 9:934-946 
Hair, J. F. (2009) Multivariate data analysis: A Global Perspective. 7th ed. Prentice Hall, Upper Saddle River:

Hurt C, Bhattacharyya S, Burns A, et al (2008) Patient and caregiver perspectives of quality of life in dementia. Dementia and geriatric cognitive disorders 26:138-146

Kumfor F, Zhen A, Hodges JR, et al (2018) Apathy in Alzheimer's disease and frontotemporal dementia: distinct clinical profiles and neural correlates. Cortex 103:350-359

Lansdall CJ, Coyle-Gilchrist ITS, Vázquez Rodríguez P, et al (2019) Prognostic importance of apathy in syndromes associated with frontotemporal lobar degeneration. Neurology 92:e1547-e1557. https://doi.org/10.1212/WNL.0000000000007249

Laplane D, Dubois B (2001) Auto-activation deficit: a basal ganglia related syndrome. Movement Disorders 16:810-814

Le Heron C, Apps. MAJ, Husain M (2018) The anatomy of apathy: A neurocognitive framework for amotivated behaviour. Neuropsychologia 118:54-67. https://doi.org/10.1016/j.neuropsychologia.2017.07.003

Lechowski L, Benoit M, Chassagne P, et al (2009) Persistent apathy in Alzheimer's disease as an independent factor of rapid functional decline: the REAL longitudinal cohort study. International Journal of Geriatric Psychiatry: A journal of the psychiatry of late life and allied sciences $24: 341-346$

Levy R (2012) Apathy: A pathology of goal-directed behaviour. A new concept of the clinic and pathophysiology of apathy. Revue Neurologique 168:585-597. https://doi.org/10.1016/j.neurol.2012.05.003

Levy R, Dubois B (2006) Apathy and the Functional Anatomy of the Prefrontal Cortex-Basal Ganglia Circuits. Cerebral Cortex 16:916-928. https://doi.org/10.1093/cercor/bhj043 
Mak LE, Minuzzi L, MacQueen G, et al (2017) The Default Mode Network in Healthy Individuals: A Systematic Review and Meta-Analysis. Brain Connectivity 7:25-33. https://doi.org/10.1089/brain.2016.0438

Malpetti M, Jones PS, Tsvetanov KA, et al (2021) Apathy in presymptomatic genetic frontotemporal dementia predicts cognitive decline and is driven by structural brain changes. Alzheimer's \& Dementia 17:969-983. https://doi.org/10.1002/alz.12252

Marin RS (1996) Apathy: Concept, Syndrome, Neural Mechanisms, and Treatment. In: Seminars in clinical neuropsychiatry. pp 304-314

Marin RS (1991) Apathy: a neuropsychiatric syndrome. The Journal of neuropsychiatry and clinical neurosciences 3:243-254. https://doi.org/10.1016/j.neuropsychologia.2017.07.003

Marin RS, Biedrzycki RC, Firinciogullari S (1991) Reliability and validity of the apathy evaluation scale. Psychiatry Research 38:143-162. https://doi.org/10.1016/01651781(91)90040-V

Massimo L, Evans LK, Grossman M (2014) Differentiating subtypes of apathy to improve person-centered care in frontotemporal degeneration. Journal of gerontological nursing $40: 58-65$

Massimo L, Powers JP, Evans LK, et al (2015) Apathy in Frontotemporal Degeneration: Neuroanatomical Evidence of Impaired Goal-directed Behavior. Frontiers in Human Neuroscience 9:. https://doi.org/10.3389/fnhum.2015.00611

Mattis S (1976) Mental status examination for organic mental syndrome in the elderly patients. In Bellack L, Karasu TB (eds.) Geriatric Psychiatry A Hand Book for Psychiatrics and Primary Care Physicians. Grune and Stratton, New York, pp 77-121.

Mattis S (1988) Dementia rating scale: professional manual. Psychological Assessment 
Resources, Incorporated

Pasquier F, Lebert F, Lavenu I, Guillaume B (1999) The Clinical Picture of Frontotemporal

Dementia: Diagnosis and Follow-Up. DEM 10:10-14. https://doi.org/10.1159/000051206

Radakovic R, Abrahams S (2014) Developing a new apathy measurement scale: Dimensional Apathy Scale. Psychiatry research 219:658-663

Radakovic R, Colville S, Cranley D, et al (2021) Multidimensional Apathy in Behavioral Variant Frontotemporal Dementia, Primary Progressive Aphasia, and Alzheimer Disease. J Geriatr Psychiatry Neurol 34:349-356. https://doi.org/10.1177/0891988720924716

Raichle ME, MacLeod AM, Snyder AZ, et al (2001) A default mode of brain function.

Proceedings of the National Academy of Sciences 98:676-682

Rascovsky K, Hodges JR, Kipps CM, et al (2007) Diagnostic Criteria for the Behavioral Variant of Frontotemporal Dementia (bvFTD): Current Limitations and Future Directions:

Alzheimer Disease \& Associated Disorders 21:S14-S18.

https://doi.org/10.1097/WAD.0b013e31815c3445

Rascovsky K, Hodges JR, Knopman D, et al (2011) Sensitivity of revised diagnostic criteria for the behavioural variant of frontotemporal dementia. Brain 134:2456-2477. https://doi.org/10.1093/brain/awr179

Raubenheimer J (2004) An item selection procedure to maximise scale reliability and validity. SA j ind psychol 30:. https://doi.org/10.4102/sajip.v30i4.168

Robert P, Lanctôt KL, Agüera-Ortiz L, et al (2018) Is it time to revise the diagnostic criteria for apathy in brain disorders? The 2018 international consensus group. European Psychiatry 54:71-76. https://doi.org/10.1016/j.eurpsy.2018.07.008

Schmidt L, d'Arc BF, Lafargue G, et al (2008) Disconnecting force from money: effects of 
basal ganglia damage on incentive motivation. Brain 131:1303-1310.

https://doi.org/10.1093/brain/awn045

Schroeter ML, Vogt B, Frisch S, et al (2011) Dissociating behavioral disorders in early dementia — an FDG-PET study. Psychiatry Research: Neuroimaging 194:235-244

Shaw SR, El-Omar H, Roquet D, et al (2021) Uncovering the prevalence and neural substrates of anhedonia in frontotemporal dementia. Brain 144:1551-1564. https://oi.org/10.1093/brain/awab032

Starkstein SE, Mayberg HS, Preziosi T, et al (1992) Reliability, validity, and clinical correlates of apathy in Parkinson's disease. J Neuropsychiatry Clin Neurosci 4:134-139 Sun H-H, Pan P-L, Hu J-B, et al (2020) Alterations of regional homogeneity in Parkinson's disease with "pure" apathy: a resting-state fMRI study. Journal of Affective Disorders Tabachnick BG, Fidell LS (2007) Using multivariate statistics, 5th edn. Allyn and Bacon, Boston.

Tumati S, Marsman J-BC, De Deyn PP, et al (2020) Functional network topology associated with apathy in Alzheimer's disease. Journal of Affective Disorders 266:473-481

Tumati S, Martens S, de Jong BM, Aleman A (2019) Lateral parietal cortex in the generation of behavior: Implications for apathy. Progress in Neurobiology 175:20-34. https://doi.org/10.1016/j.pneurobio.2018.12.003 van den Heuvel MP, Hulshoff Pol HE (2010) Exploring the brain network: A review on resting-state fMRI functional connectivity. European Neuropsychopharmacology 20:519-534. https://doi.org/10.1016/j.euroneuro.2010.03.008

Wadsworth LP, Lorius N, Donovan NJ, et al (2012) Neuropsychiatric symptoms and global functional impairment along the Alzheimer's continuum. Dementia and geriatric cognitive 
disorders 34:96-111

Wei G, Irish M, Hodges JR, et al (2020) Disease-specific profiles of apathy in Alzheimer's disease and behavioural-variant frontotemporal dementia differ across the disease course. $\mathrm{J}$ Neurol 267:1086-1096. https://doi.org/10.1007/s00415-019-09679-1

Whitfield-Gabrieli S, Nieto-Castanon A (2012) Conn: a functional connectivity toolbox for correlated and anticorrelated brain networks. Brain connectivity 2:125-141

Yuen GS, Gunning-Dixon FM, Hoptman MJ, et al (2014) The salience network in the apathy of late-life depression. International journal of geriatric psychiatry 29:1116-1124

Zhou J, Greicius MD, Gennatas ED, et al (2010) Divergent network connectivity changes in behavioural variant frontotemporal dementia and Alzheimer's disease. Brain 133:1352-1367. https://doi.org/10.1093/brain/awq075

Zhou J, Seeley WW (2014) Network Dysfunction in Alzheimer's Disease and Frontotemporal Dementia: Implications for Psychiatry. Biological Psychiatry 75:565-573.

https://doi.org/10.1016/j.biopsych.2014.01.020

Zhou Z, Zheng X, Li R, et al (2020) Alterations of Cerebral Blood Flow Network in Behavioral Variant Frontotemporal Dementia patients with and without Apathy. Psychiatry Research: Neuroimaging 111203

Zou Q-H, Zhu C-Z, Yang Y, et al (2008) An improved approach to detection of amplitude of low-frequency fluctuation (ALFF) for resting-state fMRI: fractional ALFF. Journal of neuroscience methods 172:137-141 


\section{STATEMENTS AND DECLARATIONS}

\section{Funding}

This study was funded by grant ANR-10-IAIHU-06 from the program "Investissements d'avenir", by grant FRM DEQ20150331725 from the foundation "Fondation pour la recherche médicale", and by the ENEDIS company. Valérie Godefroy is supported by Malakoff Médéric Humanis company. Arabella Bouzigues is supported by "Fondation Vaincre Alzheimer". Raffaella Migliaccio is supported by France Alzheimer and Philippe Chatrier Foundations, and by Rosita Gomez association.

\section{Competing interests}

The authors report no competing interests.

\section{Author Contributions}

Study supervision: RL. Study conception and design: VG, BB, RL. Data acquisition: RM, BB, RL, DB, GC, ARL, SB, EC. Analysis and interpretation of data: VG, SC. Drafting the manuscript: VG, AB, BB, RM, RL. Obtaining funding: RL, BB. All authors critically revised the manuscript for its intellectual content.

\section{Data availability}

The data that support the findings of this study are available from the corresponding author, upon reasonable request.

\section{Ethics approval}

This study is part of clinical trial C16-87 sponsored by INSERM, the French national institute for biomedical research. It was granted approval by the local Ethics Committee ("Comité de Protection des Personnes") on 05/17/2017 and registered in a public registry (clinicaltrials.gov: NCT03272230). 


\section{Consent to participate}

All study participants gave their written informed consent to participate, according to the Declaration of Helsinki and in line with French ethical guidelines. 


\section{Supplementary Files}

This is a list of supplementary files associated with this preprint. Click to download.

- Supplementaryfile1.pdf 